\title{
Effect of Adrenomedullin Administration in Two Rat Models of Experimental Inflammatory Bowel Disease
}

\author{
Sayaka Nagata*, Tomomi Hikosaka, Kazuo Kitamura \\ Divivion of Circulatory and Body Fluid Regulation, Faculty of Medicine, University of Miyazaki, Miyazaki, Japan
}

\section{Email address:}

sayaka_nagata@med.miyazaki-u.ac.jp (S. Nagata), tomomi_hikosaka@med.miyazaki-u.ac.jp (T. Hikosaka),

kazuokit@med.miyazaki-u.ac.jp (K. Kitamura)

\section{To cite this article:}

Sayaka Nagata, Tomomi Hikosaka, Kazuo Kitamura. Effect of Adrenomedullin Administration in Two Rat Models of Experimental Inflammatory Bowel Disease. American Journal of Life Sciences. Special Issue: Biology and Medicine of Peptide and Steroid Hormones. Vol. 3, No. 3-2, 2015, pp. 39-42. doi: 10.11648/j.ajls.s.2015030302.17

\begin{abstract}
Adrenomedullin (AM) is a novel hypotensive peptide that also exerts powerful anti-inflammatory effects. We recently showed that AM significantly reduces the clinical severity of acetic acid-induced colitis, an experimental model of inflammatory bowel disease (IBD) in rats. In the present study, we examined the effect of AM in two alternative rat models of IBD. We found that 2,4,6-trinitrobenzenesulfonic acid (TNBS) induced megacolon development in the saline-treated group, but AM treatment reduced the macroscopic damage caused by TNBS. In the dextran sulfate sodium (DSS) model, treatment with AM reduced diarrhea and bloody stool scores, but did not reduce body weight. Histological analysis revealed that in both the TNBS and DSS models, colon inflammation was much more severe in the saline-treated group than in the AM-treated group. These findings indicate that the anti-inflammatory properties of AM make it an effective therapeutic agent for the treatment of IBD in rats.
\end{abstract}

Keywords: Adrenomedullin, Inflammatory Bowel Disease, TNBS, DSS

\section{Introduction}

Inflammatory bowel disease (IBD) comprises a group of immune-mediated chronic intestinal disorders that include ulcerative colitis (UC) and Crohn's disease (CD) ${ }^{1,2}$. IBD patient numbers are increasing yearly. The pathogenesis of IBD is generally accepted involving infiltration of neutrophils and overexpression of pro-inflammatory mediators, such as reactive oxygen metabolites and cytokines ${ }^{3}$. Although the exact nature of its mechanism of action is still uncertain.

Adrenomedullin (AM) is a pluripotent bioactive peptide, which was initially isolated from human pheochromocytoma in $1993^{4}$. Since its isolation, AM has been shown to be widely distributed among various organs and tissues, including the human digestive system, and to be involved in a variety of physiological functions such as vasodilatation, hormone secretion, neurotransmission, embryogenesis, wound healing, and immunoregulation ${ }^{5}$.

In our previous study, we report that $\mathrm{AM}$ reduces the severity of acetic acid-induced colitis in rats ${ }^{6}$. Experimental animal models are important tools that provide various results us to research the pathogenesis of diseases and to test emerging therapeutic strategies. Two colitis models, 2,4,6-trinitrobenzenesulfonic acid (TNBS) and dextran sodium sulfate (DSS), are canonical IBD models whereby the onset of inflammation is immediate and the procedure is relatively. Therefore, these two models have been frequently used $^{7-9}$.

The aim of this study was to examine the effect of AM administered in these two alternative IBD models.

\section{Materials and Methods}

\subsection{Animals}

Male Wistar rats at 7 weeks of age were purchased from Charles River Laboratories (Kanagawa, Japan) and maintained under a $12 \mathrm{~h}$ light $/ 12 \mathrm{~h}$ dark cycle in specific pathogen-free conditions with a normal diet. The present study was performed in accordance with the Animal Welfare Act and with the approval of the University of Miyazaki Institutional Animal Care and Use Committee (2008-501-2). 


\subsection{Peptide and Chemicals}

The recombinant human AM used in this study was provided by Sionogi (Osaka, Japan). TNBS and DSS were purchased from Nacalai Tesque (Kyoto, Japan) and Wako Pure Chemical Industries, Ltd. (Osaka, Japan), respectively.

\subsection{Induction of TNBS Colitis and Treatment with AM}

Rats were fasted for $24 \mathrm{~h}$ prior to the induction of colitis, but were freely allowed tap water throughout. TNBS colitis was induced by rectal administration of TNBS (30 mg in 50\% ethanol, $0.5 \mathrm{~mL}$ total volume) via a polyethylene catheter inserted $8 \mathrm{~cm}$ from the anus. Following instillation, the animals were maintained in a head-down position for 30 seconds to prevent leakage. AM $(1.0 \mu \mathrm{g} / \mathrm{hr} / \mathrm{rat})$ or saline (control) were given to rats on the start day of TNBS application via the intravenous route using osmotic mini pumps (ALZET Osmotic Pumps, Cupertino, CA, USA) and continued until day 14. Rats were sacrificed on day 14 after induction of colitis. In case of died before sacrifice, we performed an autopsy (Fig. 2). An overview of the experimental design is shown in Fig. 1.

\subsection{Induction of DSS Colitis and Treatment with AM}

DSS colitis was induced by an intake of $5 \%(\mathrm{w} / \mathrm{v})$ DSS $(5,000 \mathrm{MW})$ dissolved in drinking water for 3 consecutive days, which was then was replaced with $1 \%$ DSS water for 11 days. AM $(5.0 \mu \mathrm{g} / \mathrm{hr} / \mathrm{rat})$ or saline (control) were given to rats on the start day of DSS application via the intravenous route using osmotic mini pumps and continued until day 14. Rats were sacrificed on day 14 after induction of colitis. An overview of the experimental design is shown in Fig. 1.

\section{A. TNBS colitis}
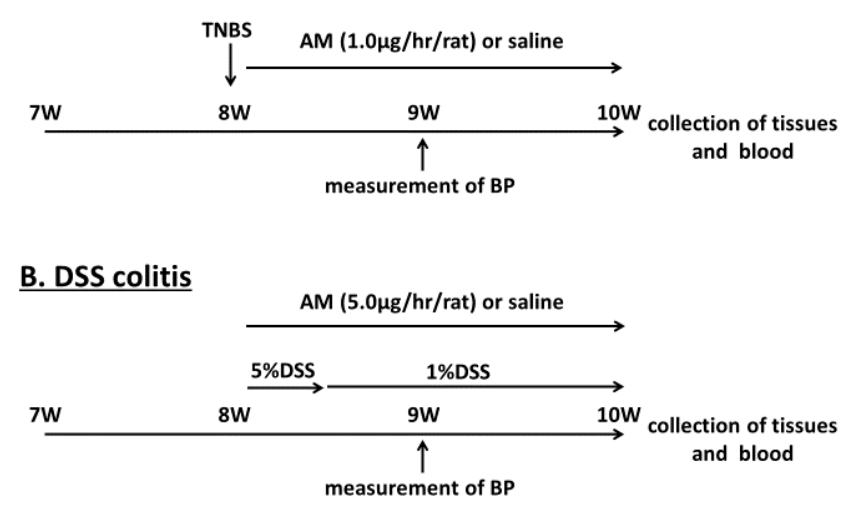

Figure 1. Overview of experimental design. (A) TNBS colitis model. (B) DSS colitis model.

\subsection{Score of Diarrhea and Bloody Stool}

Rats were checked daily for body weight, diarrhea and stool condition. Scores of diarrhea and bloody stool are shown table1.

\subsection{Histological Analysis}

Samples of colonic tissue were fixed in buffered $10 \%$ formaldehyde. Sections $(5 \mu \mathrm{m})$ were stained with hematoxylin and eosin, then processed for histological examination using standard techniques ${ }^{6}$.

\subsection{Statistical Analysis}

Comparisons of all data were made employing analysis of student T-tests. Values are presented as means \pm S.E., and significance was set at $\mathrm{P}<0.05$.

Table 1. Score of Diarrhea and Bloody Stool

\begin{tabular}{lll}
\hline Score & Diarrhea & Bloody stool \\
\hline 1 & Normal & No blood \\
2 & Soft with well-formed pellets & Feces spotted with blood \\
3 & Soft without pellets & Faces stained with blood \\
4 & Diarrhea & Gross bleeding \\
\hline
\end{tabular}

\section{Results}
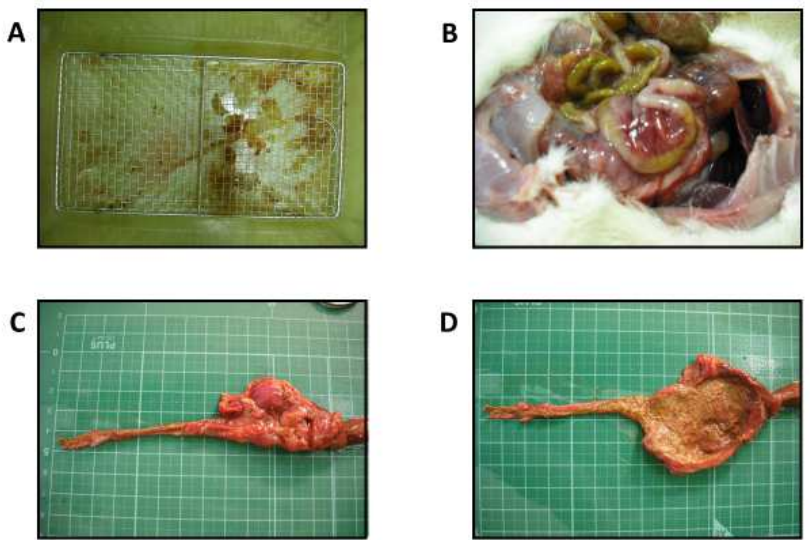

Figure 2. (A) The rat cage in which ileus was caused by TNBS treatment. There was no trace of emptied bowels for seven days. (B) Small amount of abdominal dropsy and the intestinal tracts stuck together. (C) The large intestine was removed and is shown to have formed a megacolon. (D) Mucous membranes were tattered following colitis induction.
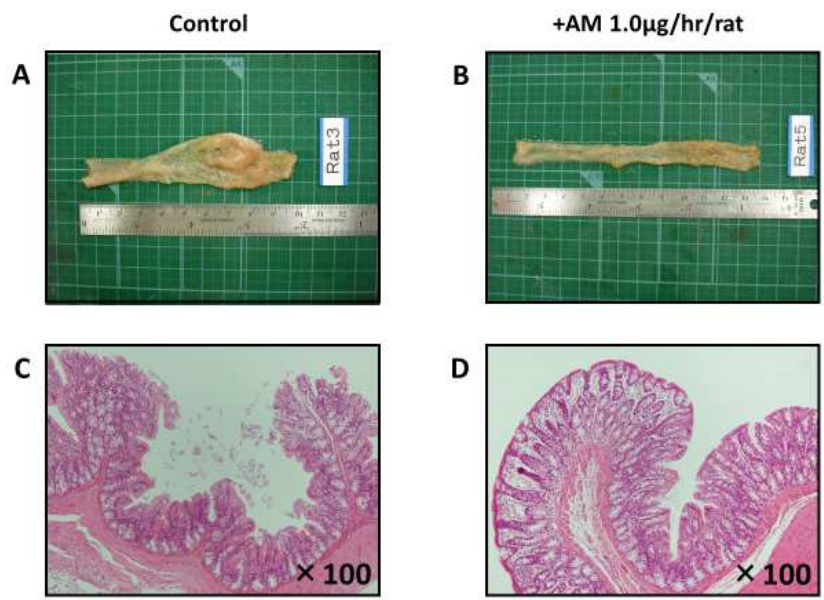

Figure 3. Representative colon appearance in the (A) saline and (B) AM-treated groups of TNBS colitis model. Representative histological appearance of rat colonic mucosa in the (C) saline and (D) AM-treated groups. 
We performed two experiments as shown Fig. 1. In the TNBS colitis model (Fig. 1A), body weight and colon length did not differ between both groups (data not shown). In addition, no significant differences were noted in systolic and diastolic blood pressures (SBP and DBP, respectively) between both groups (data not shown). TNBS treatment induced megacolon and ileus development prior to death in the saline-treated group (Fig. 2A-D). In the AM treated group, megacolon and ileus development were not observed. Histological analysis revealed that in the TNBS model, colon inflammation was much more severe in the saline-treated group than in the AM-treated group (Fig. 3A-D).
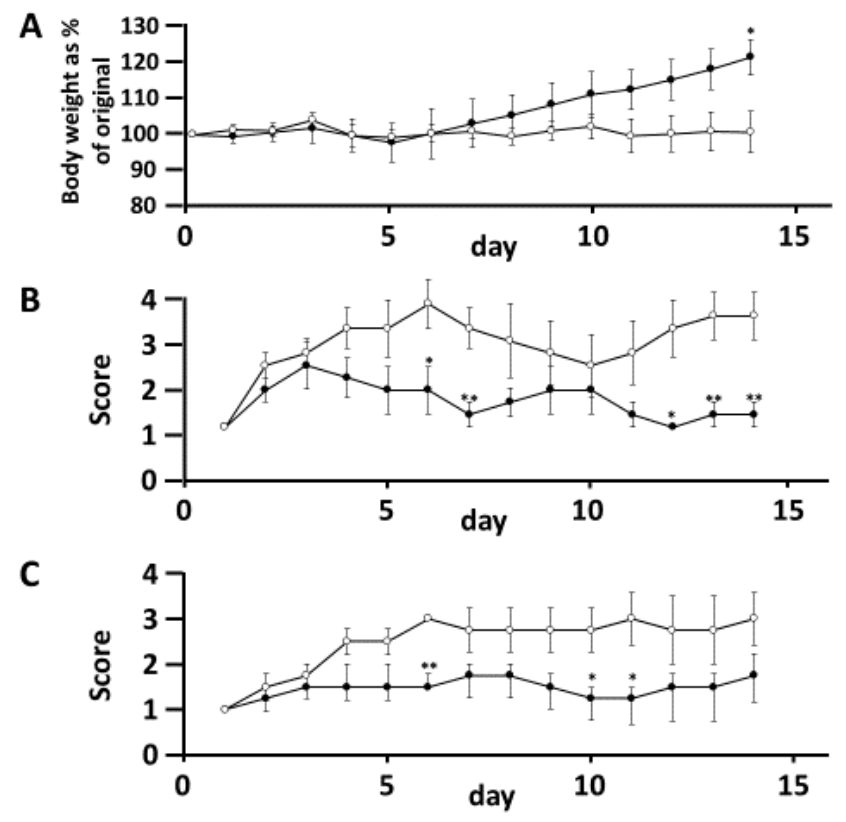

Figure 4. Daily changes of body weight (A), score of diarrhea (B) and score of bloody stool (C) in DSS colitis model: closed circles, AM-treated groups; open circles, saline groups. The results are shown as means $\pm S . E(n=4)$. ${ }^{*} p<0.05,{ }^{* *} p<0.02$ vs. control.
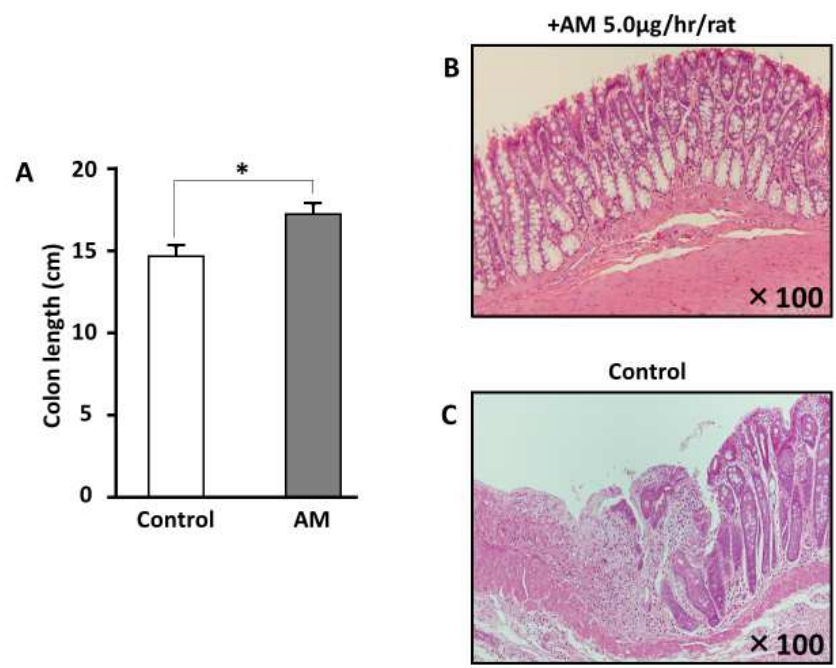

Figure 5. Comparison of (A) colon length (cm) and $(B-C)$ histology in DSS colitis model. The results are shown as mean \pm S.E. $(n=4)$. $*^{*}<0.05$ vs. control.
In the DSS colitis model (Fig. 1B), treatment with AM increased body weight (Fig. 4A) and reduced diarrhea (Fig. 4B) and bloody stool scores (Fig. 4C). No significant differences were noted in SBP and DBP (data not shown). Furthermore, colon length in the AM-treated group was longer than in the saline-treated group (Fig. 5A). Histological analysis revealed that in the DSS model, colon inflammation was much more severe in the saline-treated group than in the AM-treated group (Fig. 5B, C).

\section{Discussion}

In this report, we describe the effect of AM treatment in two alternative rat models of IBD. AM, isolated from human pheochromocytoma tissues arising from the adrenal medulla, is a biologically active peptide with multiple effects, including powerful vasodilating action, and is now known to exert a wide range of physiological effects, including cardiovascular protection, neovascularization and apoptosis suppression ${ }^{4,5,10}$. In this study, no significant differences were noted in SBP and DBP in both models. Therefore AM did not have an adverse effect on blood pressure and could be a suitable candidate IBD therapeutic.

AM, amylin and calcitonin gene-related peptide (CGRP) show close sequence homology and present overlapping spectra of biological activity. The CGRP superfamily can influence gastrointestinal activities through the autonomic nervous system ${ }^{11,12}$. In addition, some evidence shows that AM can elicit a gastroprotective effect on acetic acid- ${ }^{6}$, ethanol- ${ }^{12}$ and reserpine-induced gastric lesions models ${ }^{13}$. In the present study, we demonstrated that AM can also elicit a gastroprotective effect on two alternative models. Our findings coincide with these reports.

TNBS treatment induced megacolon development in the saline-treated group, while AM treatment reduced the macroscopic damage caused by TNBS. In addition, histological analysis revealed that in the TNBS model, colon tissue inflammation was much more severe in the saline-treated group. The TNBS colitis model results in a more severe condition compared with the DSS colitis model. However, we were unable to find differences in body weight and colon length in the TNBS model. Because TNBS is injected only once to make this model, no difference in gross appearance was observed between the saline and AM-treated groups.

Conversely, in the DSS colitis model treated with AM, an increased body weight and colon length were observed, with reduced diarrhea and bloody stool scores. Furthermore, colon tissue inflammation was much more severe in the saline-treated group as observed by histological analysis.

In IBD and experimental colitis, blood monocytes are recruited to the mucosa where they differentiate into activated macrophages that produce pro-inflammatory cytokines, such as tumor necrosis factor- $\alpha$ (TNF $\alpha$ ), interleukin (IL)-1 and IL- $6{ }^{14,15}$. It can therefore be deduced that AM treatment inhibits production of TNF $\alpha$, IL-1 and IL-6 via suppression of activated macrophages. However, 
further investigations are needed to confirm these findings.

$\mathrm{AM}$ has been shown to be widely distributed in gastrointestinal tissues including the stomach, duodenum, jejunum, ileum, colon, and rectum ${ }^{16,17}$. AM receptor expression has also been shown to be widely distributed in many of these organs and tissues ${ }^{18,19}$. It is therefore believed that AM can act directly on these tissues.

Indeed, we are currently working on clarifying the mechanisms by which AM protects from colitis. In the present study, we demonstrated a protective effect of AM treatment against colitis induced by TNBS and DSS in rats. These findings indicate that the anti-inflammatory properties of AM make it an effective candidate therapeutic agent for the treatment of IBD. Further studies of AM treatment will expand on these findings.

\section{Acknowledgements}

This study was supported in part by Grants-in-Aid for Scientific Research from the Ministry of Education, Culture, Sports, Science and Technology of Japan, and Health and Labor Science Research Grants for Translational Research from the Ministry of Health, Labor and Welfare, Japan.

\section{References}

[1] Fiocchi, C. Inflammatory bowel disease: etiology and pathogenesis. Gastroenterology 115, 182-205 (1998).

[2] Binion, D. G., West, G. A., Volk, E. E., Drazba, J. A., Ziats, N. P., Petras, R. E. Fiocchi, C. Acquired increase in leucocyte binding by intestinal microvascular endothelium in inflammatory bowel disease. Lancet 352, 1742-1746, doi:10.1016/S0140-6736(98)05050-8 (1998).

[3] Salim, S. Y.Soderholm, J. D. Importance of disrupted intestinal barrier in inflammatory bowel diseases. Inflammatory bowel diseases 17, 362-381, doi:10.1002/ibd.21403 (2011).

[4] Kitamura, K., Kangawa, K., Kawamoto, M., Ichiki, Y., Nakamura, S., Matsuo, H. Eto, T. Adrenomedullin: a novel hypotensive peptide isolated from human pheochromocytoma. Biochemical and biophysical research communications 192, 553-560, doi:10.1006/bbrc.1993.1451 (1993).

[5] Kitamura, K., Kangawa, K. Eto, T. Adrenomedullin and PAMP: discovery, structures, and cardiovascular functions. Microscopy research and technique 57, 3-13, doi:10.1002/jemt.10052 (2002).

[6] Ashizuka, S., Ishikawa, N., Kato, J., Yamaga, J., Inatsu, H., Eto, T. Kitamura, K. Effect of adrenomedullin administration on acetic acid-induced colitis in rats. Peptides 26, 2610-2615, doi:10.1016/j.peptides.2005.05.007 (2005).

[7] Du, C., Wang, P., Yu, Y., Chen, F., Liu, J. Li, Y. Gadolinium chloride improves the course of TNBS and DSS-induced colitis through protecting against colonic mucosal inflammation. Scientific reports 4, 6096, doi:10.1038/srep06096 (2014).

[8] Jakobsson, T., Vedin, L. L., Hassan, T., Venteclef, N., Greco, D., D'Amato, M., Treuter, E., Gustafsson, J. A. Steffensen, K. R. The oxysterol receptor LXRbeta protects against DSS- and TNBS-induced colitis in mice. Mucosal immunology 7, 1416-1428, doi:10.1038/mi.2014.31 (2014).

[9] Alkahtani, R., Mahavadi, S., Al-Shboul, O., Alsharari, S., Grider, J. R. Murthy, K. S. Changes in the expression of smooth muscle contractile proteins in TNBS- and DSS-induced colitis in mice. Inflammation 36, 1304-1315, doi:10.1007/s10753-013-9669-0 (2013).

[10] Beltowski, J.Jamroz, A. Adrenomedullin--what do we know 10 years since its discovery? Polish journal of pharmacology 56, 5-27 (2004).

[11] Tache, Y. Inhibition of gastric acid secretion and ulcers by calcitonin [correction of calciton] gene-related peptide. Annals of the New York Academy of Sciences 657, 240-247 (1992).

[12] Kaneko, H., Mitsuma, T., Nagai, H., Mori, S., Iyo, T., Kusugami, K. Tache, Y. Central action of adrenomedullin to prevent ethanol-induced gastric injury through vagal pathways in rats. The American journal of physiology 274, R1783-1788 (1998).

[13] Clementi, G., Caruso, A., Cutuli, V. M., Mangano, N. G., Salomone, S., Lempereur, L., Prato, A., Matera, M. Amico-Roxas, M. Gastroprotective effect of adrenomedullin administered subcutaneously in the rat. Peptides 23, 1149-1153 (2002).

[14] Maloy, K. J.Powrie, F. Intestinal homeostasis and its breakdown in inflammatory bowel disease. Nature 474, 298-306, doi:10.1038/nature10208 (2011).

[15] Bain, C. C., Scott, C. L., Uronen-Hansson, H., Gudjonsson, S., Jansson, O., Grip, O., Guilliams, M., Malissen, B., Agace, W. W. Mowat, A. M. Resident and pro-inflammatory macrophages in the colon represent alternative context-dependent fates of the same Ly6Chi monocyte precursors. Mucosal immunology 6, 498-510, doi:10.1038/mi.2012.89 (2013).

[16] Sakata, J., Asada, Y., Shimokubo, T., Kitani, M., Inatsu, H., Kitamura, K., Kangawa, K., Matsuo, H., Sumiyoshi, A. Eto, T. Adrenomedullin in the gastrointestinal tract. Distribution and gene expression in rat and augmented gastric adrenomedullin after fasting. Journal of gastroenterology 33, 828-834 (1998).

[17] Kitani, M., Sakata, J., Asada, Y., Kitamura, K. Eto, T. Distribution and expression of adrenomedullin in human gastrointestinal tissue. Annals of clinical biochemistry $35(\mathrm{Pt}$ 5), 643-648 (1998).

[18] Hay, D. L.Smith, D. M. Adrenomedullin receptors: molecular identity and function. Peptides 22, 1753-1763 (2001).

[19] McLatchie, L. M., Fraser, N. J., Main, M. J., Wise, A., Brown, J., Thompson, N., Solari, R., Lee, M. G. Foord, S. M. RAMPs regulate the transport and ligand specificity of the calcitonin-receptor-like receptor. Nature 393, 333-339, doi:10.1038/30666 (1998). 